\title{
Applicability and performance of EUCAST's rapid antimicrobial susceptibility testing (RAST) on primarily sterile body fluids in blood culture bottles in laboratory routine with total lab automation
}

\author{
Jasmin Kaur Jasuja ${ }^{1} \cdot$ Stefan Zimmermann ${ }^{1} \cdot$ Irene Burckhardt $^{1}$ \\ Received: 25 August 2020 / Accepted: 28 December 2020 / Published online: 12 January 2021 \\ (C) The Author(s) 2021
}

\begin{abstract}
Optimisation of microbiological diagnostics in primarily sterile body fluids is required. Our objective was to apply EUCAST's RAST on primarily sterile body fluids in blood culture bottles with total lab automation (TLA) and to compare results to our reference method Vitek2 in order to report susceptibility results earlier. Positive blood culture bottles (BACTEC ${ }^{\text {TM }}$ Aerobic/ Anaerobic/PEDS) inoculated with primarily sterile body fluids were semi-automatically subcultured onto Columbia 5\% SB agar, chocolate agar, MacConkey agar, Schaedler/KV agar and Mueller-Hinton agar. On latter, cefoxitin, ampicillin, vancomycin, piperacillin/tazobactam, meropenem and ciprofloxacin were added. After $6 \mathrm{~h}$, subcultures and RAST were imaged and MALDITOF MS was performed. Zone sizes were digitally measured and interpreted following RAST breakpoints for blood cultures. MIC values were determined using Vitek2 panels. During a 1-year period, 197 Staphylococcus aureus, 91 Enterococcus spp., 38 Escherichia coli, 11 Klebsiella pneumoniae and 8 Pseudomonas aeruginosa were found. Categorical agreement between RAST and MIC was $96.5 \%$. Comparison showed no very major errors, $2 / 7$ (28.6\%) and 1/7 (14.3\%) of major errors for P. aeruginosa and meropenem and ciprofloxacin, 1/9 (11.1\%) for K. pneumoniae and ciprofloxacin, 4/69 (7.0\%) and 3/43 (5.8\%) for Enterococcus spp. and vancomycin and ampicillin, respectively. Minor errors for P. aeruginosa and meropenem $(1 / 8 ; 12.8 \%)$ and for E. coli and ciprofloxacin $(2 / 29 ; 6.5 \%)$ were found. 30/550 RAST measurements were within area of technical uncertainty. RAST is applicable and performs well for primarily sterile body fluids in blood culture bottles, partially better than blood-based RAST. Official EUCAST evaluation is needed.
\end{abstract}

Keywords RAST $\cdot$ EUCAST $\cdot$ Primarily sterile body fluids $\cdot$ Blood cultures $\cdot$ TLA

\section{Introduction}

The improved diagnosis of causative pathogens in primarily sterile body fluids is an important but difficult goal to achieve in the microbiological field. Gram staining from native specimens is often non-contributory and even standard cultivation can miss microbial organisms despite specific clinical signs $[1,2]$. For this, the addition of primarily sterile body fluids, e.g. joint, pleural or peritoneal fluids, to blood culture media

Jasmin Kaur Jasuja

jasmin.jasuja@med.uni-heidelberg.de

1 Department for Infectious Diseases, University Hospital Heidelberg, Im Neuenheimer Feld 324, 69120 Heidelberg, Germany has significantly improved and accelerated the yield of causative pathogens $[2,3]$. Furthermore, poly-microbial infections particularly with methicillin-resistant Staphylococcus aureus (MRSA), P. aeruginosa and Enterococcus spp. are diagnosed with higher sensitivity [3]. Indeed, such pathogens are of special clinical relevance regarding appropriate antimicrobial therapy. As Zelenitsky et al. showed most common and significant organisms causing peritoneal-dialysis-related peritonitis like S. aureus, E. coli and K. pneumoniae have increased resistance patterns against commonly used antibiotics such as methicillin and ciprofloxacin compared to former elicitation [4]. Even Kitterer et al. demonstrated rising resistance leading to a change in the choice of first line therapy [5]. This is why rapid ID and rapid antimicrobial susceptibility testing (RAST) is of significant interest, even for primarily sterile body fluids. Tian et al. performed rapid microbial identification via 
MALDI-TOF MS and rapid multiple AST i.a. directly from positive primarily sterile body fluids inoculated in blood culture medium, but correct ID for Gram-positive bacteria was only achieved in $87.2 \%$ [6]. Though rapid multiple AST via Vitek AST system was successful, the average time to report was $\geq 8 \mathrm{~h}$, which is incongruent to the definition of rapid AST [6,7].

We already successfully implemented EUCAST's RAST on positive blood culture bottles with total lab automation (TLA, BD Kiestra ${ }^{\mathrm{TM}}$ ) in our laboratory routine [8]. In the current study, we investigated the applicability and performance of EUCAST's RAST on primarily sterile body fluids inoculated in blood culture bottles with TLA in clinical practice. Our aim was to explore if EUCAST's RAST is applicable on primarily sterile body fluids by comparing non-bloodbased RAST results with our routine reference Vitek2 to check if categorical results can be reported earlier by RAST and appropriate antibiotics can be applied in time.

\section{Material and methods}

\section{Settings}

The study was performed between 1st November 2018 and 30th November 2019 at the Department for Infectious Diseases at the University Hospital Heidelberg, Germany. Our analysis included BD BACTEC TM Plus Aerobic/F, BD BACTEC $^{\text {TM }}$ Plus Anaerobic/F and BD BACTECTM PEDS Plus/F blood culture bottles inoculated with primarily sterile body fluids sent during the aforementioned study period. Blood culture bottles inoculated with blood were excluded. Each bottle was analysed individually. The following methods were introduced during the study period and since then performed routinely. After arrival at our laboratory, aerobic or PEDS blood culture bottles were inoculated with $2 \mathrm{ml}$ of BD BACTEC $^{\text {TM }}$ FOS Kit and incubated in the BD BACTEC ${ }^{\text {TM }}$ FX instrument for up to 5 days or until they signalled positive [9]. Joint fluid was regularly incubated for 14 days or until flagged as positive.

Each positive bottle was processed in the semi-automatic part of our TLA by simultaneous Gram staining, subculturing and preparing RAST. Gram slides were prepared and stained manually and examined under microscope by a physician. Microscope results were sent as preliminary electronic report to the ward.

Subcultures on blood agar (Columbia agar, 5\% sheep blood, BD), chocolate agar (bioMérieux), MacConkey agar (bioMérieux) and in case of an anaerobic bottle additionally on Schaedler/KV agar (5\% sheep blood, BD) were done. RAST was prepared following EUCAST's methodology for positive blood cultures bottles. Therefore, $150 \mu$ l of primarily sterile body fluid in blood culture bottle was subcultured on a
Mueller-Hinton agar (bioMérieux) and six discs of commonly used antimicrobials, namely cefoxitin ( $30 \mu \mathrm{g}, \mathrm{BD})$, ampicillin (2 $\mu \mathrm{g}, \mathrm{BD})$, vancomycin $(5 \mu \mathrm{g}, \mathrm{BD})$, piperacillin/tazobactam $(30 / 6 \mu \mathrm{g}, \mathrm{BD})$, meropenem $(10 \mu \mathrm{g}, \mathrm{BD})$ and ciprofloxacin (5 $\mu \mathrm{g}, \mathrm{BD}$ ), were applied (Fig. 1). Streaking via magnetic rolling bead technology was done by TLA. Afterwards, aerobic plates including RAST subculture were transferred to the incubators $\left(35{ }^{\circ} \mathrm{C}, \mathrm{O}_{2}\right.$ : RAST plate, $5 \% \mathrm{CO}_{2}$ : blood agar, chocolate agar, MacConkey agar) of the TLA (ReadA Compact), while the anaerobic plate was incubated in an anaerobic jar in an external incubator. All plates were automatically imaged by TLA after $6 \mathrm{~h}$ and $23 \mathrm{~h}$ (latter except RAST). Anaerobic plates were viewed manually. MALDI-TOF MS (Microflex and Smart, Bruker Daltonik GmbH, Bremen), RAST reading and preparation of Vitek 2 were done with $6 \mathrm{~h}$ growth. Inhibition zones were digitally viewed, measured by positioning zone circles using TLA software and interpreted following the EUCAST RAST guidelines (version 1.0 and 1.1). An electronic report with ID and preliminary AST with RAST results was sent to the ward. On the next day, an electronic report with ID and final MIC results obtained from Vitek2 was sent.

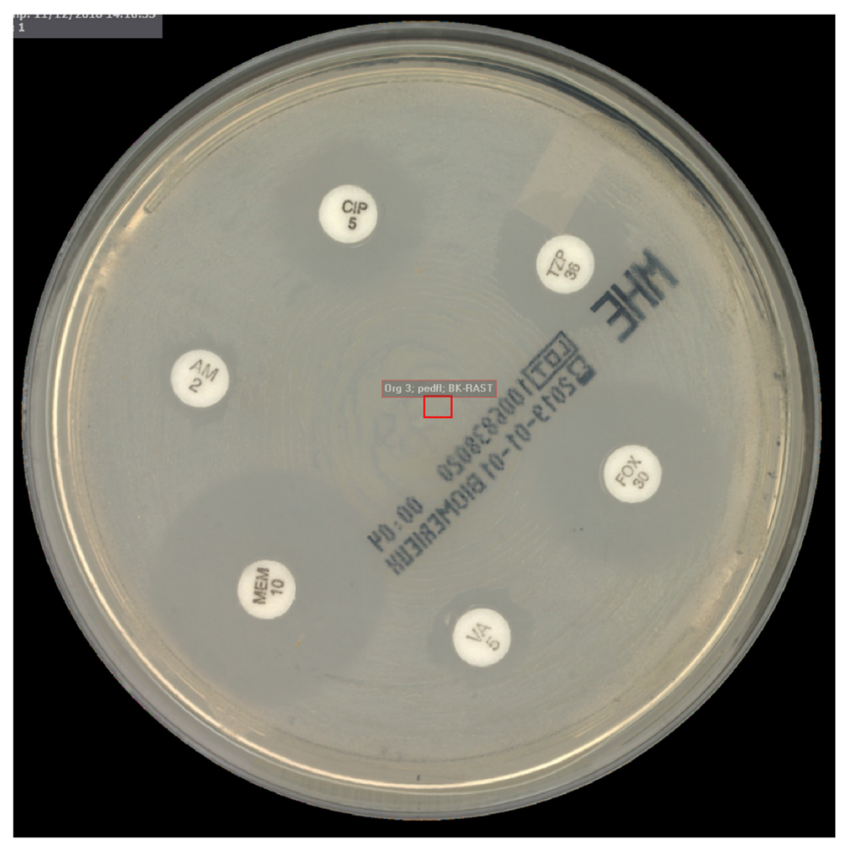

Fig. 1 RAST image of Staphylococcus aureus isolated from joint fluid after $6 \mathrm{~h}$ on Mueller-Hinton agar with visible zone diameters taken by a total lab automation (TLA) at the Department for Infectious Diseases at the University Hospital Heidelberg, Germany. As soon as a blood culture bottle inoculated with primarily sterile body fluid flagged as positive i.a. rapid antimicrobial susceptibility testing (RAST) was prepared on a Mueller-Hinton agar as established by EUCAST for blood-based RAST. After $6 \mathrm{~h}$, automatic imaging was done by TLA. Images were digitally viewed by a technician and zone diameters were measured (not measured here) (CIP, ciprofloxacin; TZP, piperacillin/tazobactam; FOX, cefoxitin; VA, vancomycin; MEM, meropenem; AM, ampicillin) 
In case of blood culture bottle signalling positivity in the late afternoon, images of subcultures and RAST were taken outside operational time ( 7 am- 6 pm on weekdays and 7 am-4 pm on weekends) and were interpreted in the next morning after performing MALDI-TOF MS. RAST was electronically reported afterwards and Vitek2 was prepared for the upcoming day.

The terms of categorical agreement, very major errors (VME), major errors (ME) and minor errors (MinE) as recommended by Cumitech were applied [10]. Originally, an error is declared as very major error (VME) when the new AST is susceptible but the reference method results in resistant response. Major errors (ME) are declared with a resistant response in the new AST while the reference method indicates a susceptible response. Minor errors (MinE) are observed when either the new AST or the reference method indicates an intermediate response and the other one a susceptible or resistant response, respectively. As we could not perform microdilution in our daily routine, we took Vitek2 as reference method. Hence, differences can also be referred as discrepancies but we continue with the generally accepted term 'error' and the recommended categories.

Since there is no intermediate category for RAST, EUCAST introduced the concept of 'area of technical uncertainty' (ATU) where interpretation to susceptible or resistant result is not possible. Hence, ATU results were not included for MinE calculation and could only arise when comparing susceptible and resistant RAST to intermediate Vitek results. Accepted percentage for categorical agreement was $\geq 90 \%$. $\mathrm{VME}$ and $\mathrm{ME}$ rate was supposed to be $\leq 3 \%$, respectively. A combined performance rate of $\leq 7 \%$ for $\mathrm{ME}$ and MinE rate was recommended. VME, ME and MinE rates were calculated for each drug and drug-species combination, respectively. For data analysis, we compared RAST with our reference method Vitek2 to check if RAST can predict final MIC results so that clinicians may adapt antimicrobial therapy earlier.

\section{Statistical analysis}

Data on RAST and MIC results were obtained from our LIS (SwissLab, Nexus AG) and analysed with Microsoft Excel 2010.

\section{Results}

During the study period from 1 st November 2018 to 30th November 2019, a total of 5341 blood culture bottles inoculated with primarily sterile body fluids were processed in our laboratory routine. Thereof, $937(17.5 \%)$ bottles signalled positive. A total of 64 bottles $(6.8 \%)$ were excluded due to poly-microbial growth which were detected with the $6 \mathrm{~h}$ growth and RAST was not reported. A total of 13 bottles $(\leq$
$1 \%$ ) were sorted out due to false-positive signal. Hence, 345 (of 860) positive mono-bacterial blood culture bottles filled with primarily sterile body fluids with readable zone diameters and available RAST breakpoints were eligible. Most of the primarily sterile body fluids contained joint fluid ( $n=$ $223)$, ascites $(n=52)$ and dialysate $(n=22)$. A total of 515 bottles contained pathogens, which do not yet have EUCAST RAST breakpoint criteria; for further analysis, see Table 1.

For 345 bottles the categorical interpretations (susceptible/ resistant) of RAST were compared to the respective Vitek2 results (susceptible/susceptible, increased exposure/resistant) (Table 2). That included 197 Staphylococcus aureus, 91 Enterococcus spp., 38 Escherichia coli, 11 Klebsiella pneumoniae and 8 Pseudomonas aeruginosa and resulted in 550 individual drug-species measurements (197x cefoxitin and $S$. aureus, 91x ampicillin and 91x vancomycin and Enterococcus spp., 57x piperacillin/tazobactam, ciprofloxacin and meropenem and E. coli, K. pneumoniae and $P$. aeruginosa altogether). As recommended by EUCAST, ATU was not interpreted [11].

Overall categorical agreement was $96.5 \%$. No VME was found in the RAST-MIC comparison. ME were found in $5.8 \%$ (4/69) and 7.0\% (3/43) for Enterococcus spp. and vancomycin and ampicillin, $11.1 \%(1 / 9)$ and $14.3 \%$ (1/7) for $K$. pneumoniae and $P$. aeruginosa and ciprofloxacin, respectively. $28.6 \%$ (2/7) for $P$. aeruginosa and meropenem. $12.5 \%$ $(1 / 8)$ and $6.5 \%(2 / 29)$ of MinE were found for P. aeruginosa and meropenem and E.coli and ciprofloxacin. Thirty out of $550(5.5 \%)$ individual drug-species measurements were ATU (non for meropenem, $28.1 \%$ for piperacillin/tazobactam and $19.3 \%$ for ciprofloxacin).

Fourteen isolates of methicillin-resistant $S$. aureus (MRSA) were found by RAST and confirmed by MIC results. The same applied to 22 vancomycin-resistant E. faecium (VRE) isolates. An isolate of $K$. pneumoniae with $b l a_{\text {OXA-48 }}$ was confirmed by PCR while RAST and MIC values reported susceptible response for meropenem (Fig. 2).

Data evaluation of drug-species combination showed no VME (Table 3). ME were found in ampicillin (7.0\%), vancomycin $(5.8 \%)$, ciprofloxacin $(4.9 \%)$ and meropenem $(4.3 \%)$. All exceeded the recommended 3\% ME rate. MinE were detected for ciprofloxacin $(4.3 \%)$ and meropenem $(1.8 \%)$. Categorical agreement for all drug-species combination exceeded the recommended $\geq 90 \%$.

\section{Discussion}

On a global scale, most sepsis deaths have an infectious cause which is why finding the source of infection is an important aim in the field of medical microbiology in order to treat properly. To do so, not only the identification of causative pathogens but also a faster and reliable AST has to be 
Table 1 Overview of all sent blood culture bottles inoculated with primarily sterile body fluids, positive signalled bottles and RASTeligible pathogens within the time period of 1st November 2018 to 30th November 2019 at the Department for Infectious Diseases at the University Hospital Heidelberg, Germany. A total of 5341 aerobic, anaerobic and PEDS blood culture bottles inoculated with various primarily sterile body fluids were sent of which 937 bottles flagged as positive. A total of 345 positive blood culture bottles were eligible for RAST and comparison to MIC results. In total, 550 drug-species measurements were analysed

Overall sent blood cultures inoculated with primarily sterile body fluids

Flagged as positive

- False-positive bottles

- Blood culture bottles with $\geq 2$ pathogens

Positive blood culture bottles with mono-bacterial growth

Pathogens for which RAST was applicable

- BACTEC ${ }^{\text {TM }}$ Aerobic

- BACTEC ${ }^{\mathrm{TM}}$ Anaerobic

- BACTEC ${ }^{\mathrm{TM}}$ PEDS

Thereof analysed drug-species measurements

Type of primarily sterile body fluids

- Joint fluid

- Ascites

- Dialysate

- Pleural fluid

- Easy flow drainage

- Other various drainage fluids (thoracic drainage, pericardium drainage, liver abscess, not-inscribed drainage)

- Gall bladder puncture

S. aureus

- MSSA

- MRSA

Enterococcus spp.

- E. faecalis

- E. faecium

- Vancomycin-resistant E. faecium (VRE) 22

E. coli

K. pneumoniae

- Carbapenemase-producing K. pneumoniae

$P$. aeruginosa

Other species than those validated for RAST 572

S. epidermidis

Other coagulase-negative Staphylococcus spp. (S. capitis, S. caprae, S. cohnii, S. haemolyticus, S. hominis, S. lugdunensis, S. petrasii,

Streptococcus spp. (S. agalactiae, S. anginosus, S. canis, S. constellatus, S. dysgalactiae, S. gallolyticus, S. gordonii, S. intermedius,

S. lutetiensis, S. mitis, S. oralis, S. parasanguinis, S. pyogenes, S. salivarius, S. sanguinis, S. thermophiles, S. vestibularis, S. pneumoniae, S. viridans)

Other Enterococcus spp. (E. gallinarum, E. avium)

Other gram-positive cocci (Aerococcus viridans, Micrococcus luteus, Parvimonas micra, Peptoniphilus harei, Rothia (Stomatococcus)

mucilaginosa, Ruminococcus gnavus)

Gram-positive bacilli (Actinomyces funkei, Actinomyces neuii, Arthrobacter sanguinis, Bacillus cereus complex, Bacillus spp., Brevibacterium 54 paucivorans, Clostridium spp., Corynebacterium spp., Cutibacterium spp., Gordonia polyisoprenivorans, Lactobacillus rhamnosus, Nocardia farcinica, Paenibacillus phoenicis)

Other Enterobacterales (Citrobacter spp., Enterobacter cloacae complex, Hafnia alvei, Klebsiella spp., Morganella morganii, Proteus spp., 7 Providencia stuartii, Serratia spp.)

Other gram-negative pathogens (Acinetobacter spp., Aeromonas caviae, Bacteroides fragilis, Burkholderia cepacia complex, Haemophilus influenzae, Moraxella spp., Pantoea spp., Pseudomonas alcaligenes)

Candida spp. 


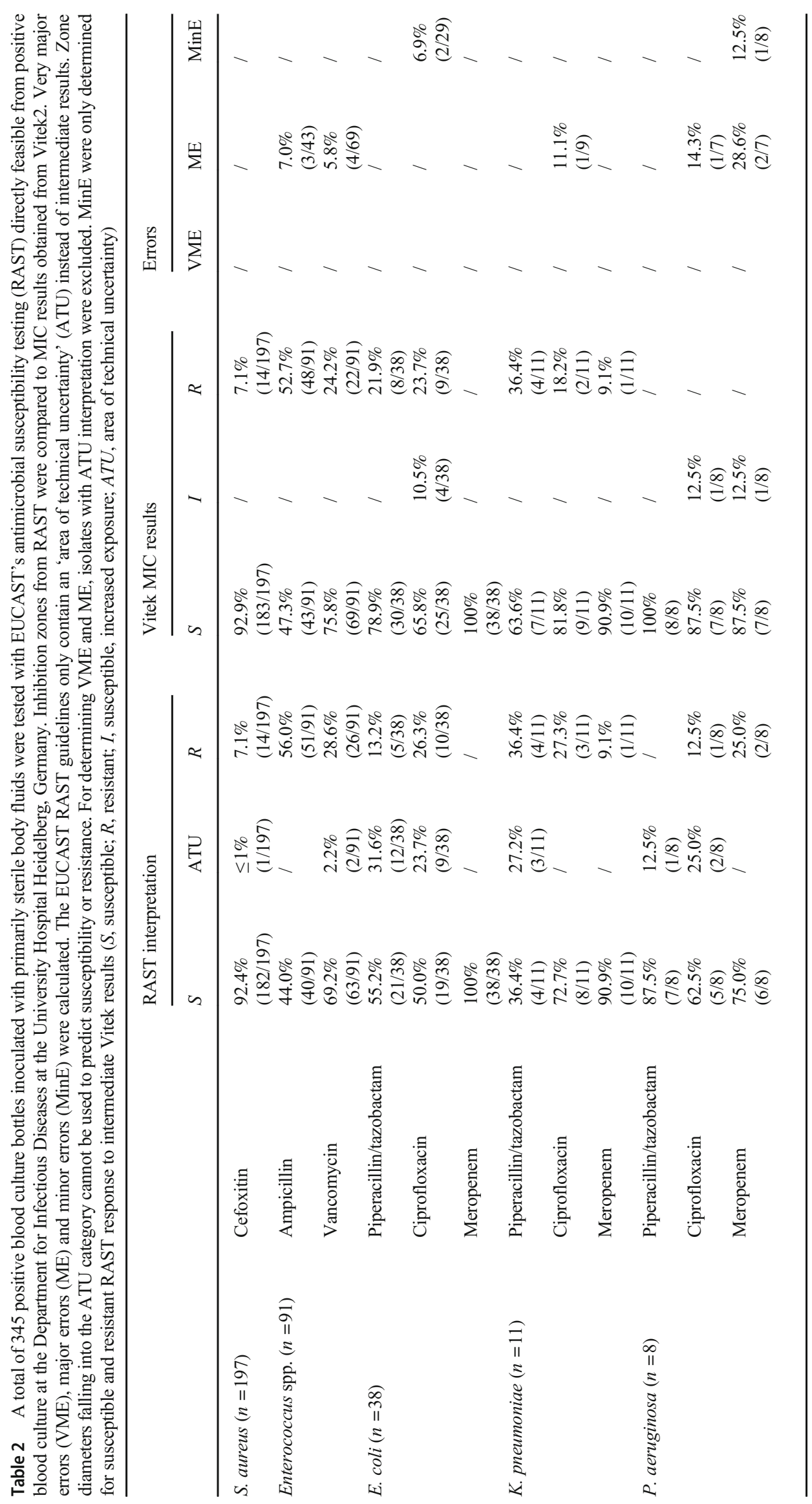




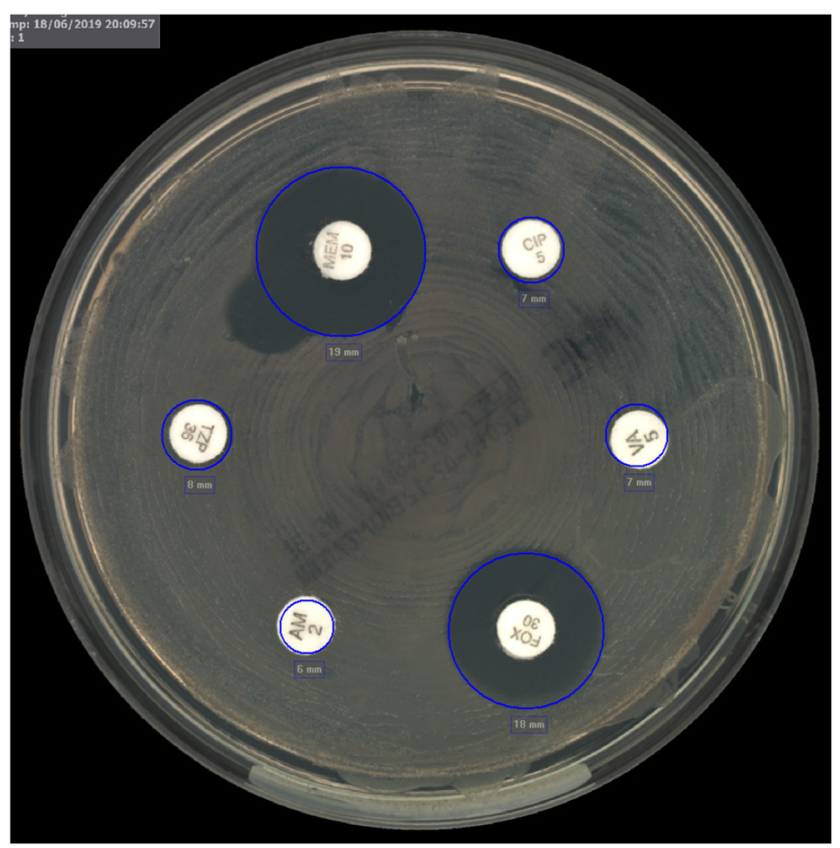

Fig. 2 RAST image of bla ${ }_{O X A-48}$ carbapenemase-producing $K$. pneumoniae in drainage fluid inoculated in blood culture bottle at the Department for Infectious Diseases at the University Hospital Heidelberg, Germany. With a zone diameter of $19 \mathrm{~mm}$, meropenem is susceptible according to the clinical breakpoints for RAST (version 1.1). MIC value of $1 \mathrm{mg} / \mathrm{L}$ obtained from Vitek 2 confirmed the susceptible RAST result. bla ${ }_{O X A-48}$ carbapenemase was detected by PCR. The growth-free area outside the zone diameter of meropenem was due to manually correction of the antimicrobial plate after stamping the disks (CIP, ciprofloxacin; TZP, piperacillin/tazobactam, FOX, cefoxitin; VA, vancomycin; MEM, meropenem; AM, ampicillin)

provided. Our current study focused on the rapid reporting of ID and AST by applying EUCAST's RAST on primarily sterile body fluids sent in a blood culture bottle to see if RAST is applicable and can predict our final results.

As shown, we did not obtain any VME, only $11 \mathrm{ME}$ and 3 MinE among 520 interpretable measurements. Though our species related errors exceeded the suggested rates by Cumitech, low denominators particularly regarding gramnegative rods like $P$. aeruginosa have to be considered. Similar problems with low denominators on blood-based blood culture bottles were recently discussed in a study on RAST by Soo et al. [12]. Furthermore, a larger number of isolates particularly more drug-resistant isolates would be useful to further evaluate the RAST method. Except the aforementioned problem with low denominators, the noninterpretation of ATU has to be considered for high species related errors as well. As already discussed by Jonasson et al. an unavoidable variation exists due to early reading, which is buffered by ATU and reduces VMEs and MEs [11]. Since challenging isolates for the establishment of RAST were used by EUCAST, our ATU fraction may be smaller due to the low level of multi-drug resistance [11]. A recent RAST study on Enterobacteriaceae by Martins et al. in Brazil showed an overall ATU of $21.6 \%$ for piperacillin/tazobactam and $5.3 \%$ for ciprofloxacin, respectively, which is lower compared to our current results [13]. Only meropenem (5.1\%) displayed a clearly higher ATU fraction while our study did not have any ATUs in meropenem. If considered, that Brazil is expected to have a higher resistance level particularly in carbapenems, this result is comprehensible. Despite, the comparison is limited as only E. coli and Klebsiella spp. were considered in the study by Martins et al., while our study included $P$. aeruginosa. Furthermore, it remains unclear, if other species except $K$. pneumoniae were considered in Martins et al. study since the study mentions Klebsiella spp., though RAST is only accredited for K. pneumoniae [13, 14]. Also, EUCAST has evaluated a delayed RAST of up to $3 \mathrm{~h}$ for positive blood cultures kept at room temperature. Martins et al. considered RAST results with a delay of $4 \pm 1 \mathrm{~h}[13,14]$.

However, the $6 \mathrm{~h}$ reading is of limited benefit if a major part of zone diameters falls into ATU which particularly regards to settings of high ESBL prevalence [15]. In fact, our study revealed an ATU fraction of $28.1 \%$ for piperacillin/tazobactam and $19.3 \%$ for ciprofloxacin which is less compared to the study by Soo et al. [12]. Despite, ATU are not reported. Hence, clinician's antimicrobial choice and a potential switch solely depend on the patient's symptoms and laboratory results meaning a great loss of the RAST intention. Compared to our blood-based RAST study both antimicrobials have less errors and ATU leading to the presumption that blood may hamper appropriate reading or growth of gram-negative pathogens [8]. Results for Grampositive cocci were similar for both studies. To our knowledge, no study on pathogen-blood interaction exists yet and consequently such a probable interaction remains interesting.

To reduce errors, EUCAST has recently updated the clinical breakpoints for $P$. aeruginosa and piperacillin/tazobactam and ciprofloxacin, respectively, by raising susceptible breakpoints to $\geq 50 \mathrm{~mm}$. Zone diameters greater than ATU $(13-15 \mathrm{~mm})$ but smaller than susceptible are suggested to be interpreted as 'susceptible, increased exposure' [16]. However, with that EUCAST correction ME rates, which were more frequent not only in our blood and non-bloodbased RAST studies, but also in studies conducted by Martins et al. and Soo et al., are not improved [12, 13]. Hence, an adaption of the resistant instead of susceptible zone diameters may rather address the problem.

Another error-prone fact was the measurement of zone diameters by numerous technicians. Though software was used, manual measurement with a difference of only $1 \mathrm{~mm}$ may lead to S/ATU/R. To minimize the observer variance, reading by a single experienced technician could be introduced which was done in a recent study with TLA [17]. However, this method is not feasible in our laboratory routine so that automatic inhibition zone reading could be a possibility. Though, automatic reading by OSIRIS system led to a slightly lower overall agreement and was additionally hampered by a poor growth particularly for 


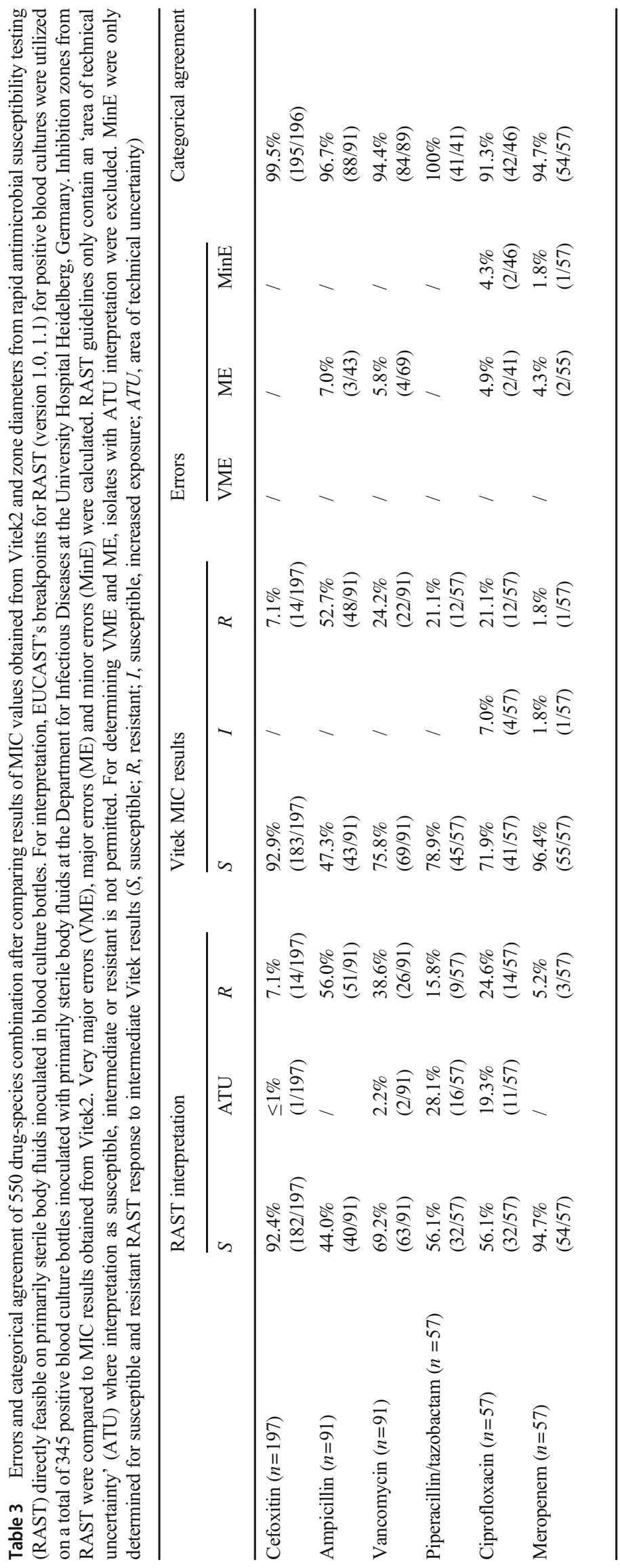


enterococci compared to manual reading [18]. Indeed, light growth and unreadable zone diameters also occurred in a study conducted by CLSI, where $92.3 \%$ of $P$. aeruginosa were unreadable after $6 \mathrm{~h}$ indicating that breakpoints for these species may not be appropriate for an early read [19]. However, it is supposed that the use of a smart incubator system like TLA increases the readability of short-incubation disk diffusion method which is why we seldom had the problem of illegibility [19]. In the current study, only $4 / 20$ (20.0\%) isolates of $P$. aeruginosa could not be read ( 8 isolates had to be excluded from the study due to missing antimicrobial disks and technical issues). Furthermore, it has been assumed that short-incubation zone diameters of resistant isolates were either smaller or larger compared to $18 \mathrm{~h}$ reading leading to unpredictability [15].

Variation not only in measurement but also in the inoculum size may mislead interpretation, e.g. quantitating the number of organisms present in $1.0 \mathrm{ml}$ of 10 randomly selected blood cultures resulted in an inoculum size ranging from $2 \times 10^{6}$ to $6 \times 10^{7}$, with a mean of $1.5 \times 10^{7}$ organisms $/ \mathrm{ml}$ [20]. Even a recent study attributed discrepancies between direct testing and reference disk diffusion to the various bacterial concentrations and evaluated three commercial systems spanning nearly $3 \operatorname{logs}$ [19]. Consequently, certain errors cannot be avoided. As Martins et al. have shown nicely, an increased inoculum size can only be compensated with an increased agar plate size resulting in 1:1 comparable zone diameters [13].

In our study we isolated one $b a_{O X A-48}$ carrying isolate of K. pneumoniae which could neither be detected by Vitek 2 nor by RAST. Similar results were found by Fröding et al. leading to the conclusion that carbapenemase cannot be detected sufficiently by $6 \mathrm{~h}$ reading [15]. In fact, our study highlights the well-known challenge for laboratories of detecting $b l a_{O X A-48}$ and again underlines the importance of additional molecular testing $[12,21]$.

Besides species with available clinical breakpoints for RAST we found a broad spectrum of other pathogens. Among them, Staphylococcus epidermidis $(n=211)$ was a frequent detected pathogen. Though pathogenicity of this bacterium is often unclear, we believe RAST would be beneficial for this certain pathogen or generally for coagulase-negative staphylococci. Considering this, we created a histogram comparing zone diameters from RAST to MIC results to show that breakpoints from $S$. aureus may not be applied on other staphylococci (Supplement 1).

A limitation of our study is our reference method. We used Vitek2 instead of broth microdilution because Vitek2 is the MIC determination method of our choice during the routine. Additionally, several papers already demonstrated that Vitek2 results are comparable to broth microdilution results [22-25]. Eventually, our aim was to demonstrate that RAST directly from positive blood culture bottles filled with primarily sterile body fluids are comparable to Vitek2 results so we can use RAST results for early reporting.
Another limitation was seen in poly-microbial findings (6.8\%) where RAST could not be applied and Vitek2 results after isolation had to be awaited.

The integration of RAST with primarily sterile body fluids in antibiotic stewardship programs remains of certain interest and we intend to conduct such a clinical trial.

\section{Conclusion}

Our study proved the applicability of RAST on various primarily sterile body fluids in blood culture bottles responding in a good categorical agreement between RAST-Vitek2, partially better than in blood-based RAST. This aim was supported by the optimal incubation in a TLA system leading to improved and punctual measurement of zone diameters.

Despite, an official evaluation with broth microdilution and recommendation by EUCAST is required. Our results support a buffer zone in form of ATU to avoid MEs or VMEs with further improvement of clinical breakpoints.

Supplementary Information The online version contains supplementary material available at https://doi.org/10.1007/s10096-020-04146-6.

Funding Open Access funding enabled and organized by Projekt DEAL.

\section{Compliance with ethical standards}

Conflict of interest The authors declare that they have no conflict of interest.

Ethical approval This article does not contain any studies with human participants performed by any of the authors.

Informed consent Not applicable.

Open Access This article is licensed under a Creative Commons Attribution 4.0 International License, which permits use, sharing, adaptation, distribution and reproduction in any medium or format, as long as you give appropriate credit to the original author(s) and the source, provide a link to the Creative Commons licence, and indicate if changes were made. The images or other third party material in this article are included in the article's Creative Commons licence, unless indicated otherwise in a credit line to the material. If material is not included in the article's Creative Commons licence and your intended use is not permitted by statutory regulation or exceeds the permitted use, you will need to obtain permission directly from the copyright holder. To view a copy of this licence, visit http://creativecommons.org/licenses/by/4.0/.

\section{References}

1. Alfa MJ, Degagne P, Olson N, Harding GK (1997) Improved detection of bacterial growth in continuous ambulatory peritoneal dialysis effluent by use of BacT/Alert FAN bottles. J Clin Microbiol 35(4):862-866 
2. Bobadilla M, Sifuentes J, Garcia-Tsao G (1989) Improved method for bacteriological diagnosis of spontaneous bacterial peritonitis. $\mathrm{J}$ Clin Microbiol 27(10):2145-2147

3. Menzies SM, Rahman NM, Wrightson JM et al (2011) Blood culture bottle culture of pleural fluid in pleural infection. Thorax. 66(8):658-662

4. Zelenitsky SA, Howarth J, Lagacé-Wiens P et al (2017) Microbiological trends and antimicrobial resistance in peritoneal dialysis-related peritonitis, 2005 to 2014. Perit Dial Int 37(2): $170-176$

5. Kitterer D, Latus J, Pöhlmann C et al (2015) Microbiological surveillance of peritoneal dialysis associated peritonitis: antimicrobial susceptibility profiles of a referral center in Germany over 32 years. PloS One 10(9):e0135969-e013596e

6. Tian Y, Zheng B, Wang B et al (2016) Rapid identification and multiple susceptibility testing of pathogens from positive-culture sterile body fluids by a combined MALDI-TOF mass spectrometry and Vitek susceptibility system. Front Microbiol 7:523

7. Kahlmeter G. Rapid phenotypic susceptibility testing. In: S536. ECCMID 2016; Amsterdam, Netherlands

8. Jasuja JK, Zimmermann S, Burckhardt I (2020) Evaluation of EUCAST rapid antimicrobial susceptibility testing (RAST) for positive blood cultures in clinical practice using a total lab automation. Eur J Clin Microbiol Infect Dis.

9. Nylén T, Saeedi B, Borg C et al (2013) The performance of 4 different supplements and 5 blood culture bottles types in detection of bacteria and Candida spp. in simulated sterile body fluid cultures. Diagn Microbiol Infect Dis. 77(1):1-4

10. Clark RB, Loeffelholz MJ, Tibbets RJ (2009) Cumitech 31A, verification and validation of procedures in the clinical microbiology laboratory. ASM Press.

11. Jonasson E, Matuschek E, Kahlmeter G (2020) The EUCAST rapid disc diffusion method for antimicrobial susceptibility testing directly from positive blood culture bottles. J Antimicrob Chemother

12. Soo YT, Waled SNMB, Ng S et al (2020) Evaluation of EUCAST rapid antimicrobial susceptibility testing (RAST) directly from blood culture bottles. Eur J Clin Microbiol Infect Dis

13. Martins A, Wink P, Pereira D et al (2020) Rapid antimicrobial susceptibility of Enterobacteriaceae by disk diffusion directly from blood culture bottles using the EUCAST RAST breakpoints. J Glob Antimicrob Resist. 22:637-642

14. The European Committee on Antimicrobial Susceptibility Testing. Methodoloy - EUCAST rapid antimicrobial susceptibility testing (RAST) directly from blood culture bottles. Version 1.1, 2019. http://www.eucast.org

15. Fröding I, Vondracek M, Giske CG (2016) Rapid EUCAST disc diffusion testing of MDR Escherichia coli and Klebsiella pneumoniae: inhibition zones for extended-spectrum cephalosporins can be reliably read after $6 \mathrm{~h}$ of incubation. J Antimicrob Chemother 72(4):1094-1102

16. The European Committee on Antimicrobial Susceptibility Testing. Zone diameter breakpoints for rapid antimicrobial susceptibility testing (RAST) directly from blood culture bottles. Version 1.0, 2018. http://www.eucast.org

17. van den Bijllaardt W, Buiting AG et al (2017) Shortening the incubation time for antimicrobial susceptibility testing by disk diffusion for Enterobacteriaceae: how short can it be and are the results accurate? Int J Antimicrob Agents. 49(5):631-637

18. Sánchez MA, del Saz BS, Loza E et al (2001) Evaluation of the OSIRIS video reader system for disk diffusion susceptibility test reading. Clin Microbiol Infect 7(7):352-357

19. Chandrasekaran S, Abbott A, Campeau S et al (2018) Direct-fromblood-culture disk diffusion to determine antimicrobial susceptibility of Gram-negative bacteria: preliminary report from the Clinical and Laboratory Standards Institute Methods Development and Standardization Working Group. J Clin Microbiol 56(3):e01678e01617

20. Doern GV, Scott DR, Rashad AL et al (1981) Evaluation of a direct blood culture disk diffusion antimicrobial susceptibility test. Antimicrob Agents Ch. 20(5):696-698

21. Bartolini A, Frasson I, Cavallaro A et al (2014) Comparison of phenotypic methods for the detection of carbapenem nonsusceptible Enterobacteriaceae. Gut Pathog. 6(1):13

22. Bobenchik AM, Deak E, Hindler JA et al (2017) Performance of Vitek 2 for antimicrobial susceptibility testing of Acinetobacter baumannii, Pseudomonas aeruginosa, and Stenotrophomonas maltophilia with Vitek 2 (2009 FDA) and CLSI M100S 26th Edition Breakpoints. J Clin Microbiol. 55(2):450

23. Bobenchik AM, Deak E, Hindler JA et al (2015) Performance of Vitek 2 for antimicrobial susceptibility testing of Enterobacteriaceae with Vitek 2 (2009 FDA) and 2014 CLSI Breakpoints. J Clin Microbiol 53(3):816

24. Bobenchik AM, Hindler JA, Giltner CL et al (2014) Performance of Vitek 2 for antimicrobial susceptibility testing of Staphylococcus spp. and Enterococcus spp. J ClinMicrobiol 52(2):392

25. Klare I, Bender JK, Fleige $\mathrm{C}$ et al (2019) Comparison of VITEK® 2, three different gradient strip tests and broth microdilution for detecting vanB-positive Enterococcus faecium isolates with low vancomycin MICs. J Antimicrob Chemother 74(10):2926-2929

Publisher's note Springer Nature remains neutral with regard to jurisdictional claims in published maps and institutional affiliations. 